\title{
Mission to Characterize Volatiles in Old, Cold, Permanently Shadowed Regions on the Moon
}

\author{
White Paper Submitted \\ To Decadal Survey in Planetary Science and Astrobiology 2023-2032 \\ 15 August 2020 \\ Point of Contact: \\ Dana M. Hurley, Johns Hopkins Applied Physics Laboratory (APL) \\ (Dana.Hurley@,jhuapl.edu, 240-228-9126)
}

Authors:

David T. Blewett, Joshua Cahill, Nancy Chabot, Benjamin Greenhagen, Charles Hibbitts, Rachel

Klima, David Lawrence, Kathleen Mandt, Jorge I. Núñez APL, Wes Patterson, Parvathy Prem, \& Angela Stickle, APL;

Ariel Deutsch, Brown University;

Mona Delitsky California Specialty Engineering;

Jessica Flahaut CNRS/CRPG;

George Sowers Colorado School of Mines;

Eliot Sefton-Nash European Space Research and Technology Centre (ESA/ESTEC);

Julie Brisset Florida Space Institute;

Kris Zacny Honeybee Robotics;

Shashwat Shukla Indian Institute of Technology Kanpur,

Jonathan Grandidier, Sona Hosseini, \& Yang Liu Jet Propulsion Laboratory;

Sriram Bhiravarasu \& Julie Stopar Lunar and Planetary Institute;

Donald Barker MAXD Inc:;

Leslie Gertsch Missouri S \& T;

Devanshu Jha MVJ College of Engineering;

Anthony Colaprete \& Richard Elphic NASA Ames Research Center,

Maria Banks, Mehdi Benna, William Farrell, Prabal Saxena, \& O.J. Tucker NASA Goddard Space

Flight Center,

Julie Mitchell NASA Johnson Space Center,

Debra Needham NASA Marshall Space Flight Center,

Jared Atkinson Norwegian Geotechnical Institute;

Simeon Barber Open University;

Amanda Hendrix, Georgiana Kramer, \& Matthew Siegler Planetary Science Institute;

Cesare Grava, Michael Poston, \& Kurt Retherford Southwest Research Institute;

Nandita Kumari SUNY Stonybrook;

Jessica Barnes University of Arizona;

Paul Hayne University of Colorado;

Shuai Li \& Paul Lucey University of Hawaï;

Timothy Livengood University of Maryland 


\section{Mission to Characterize Volatiles in Old, Cold, Permanently Shadowed Regions}

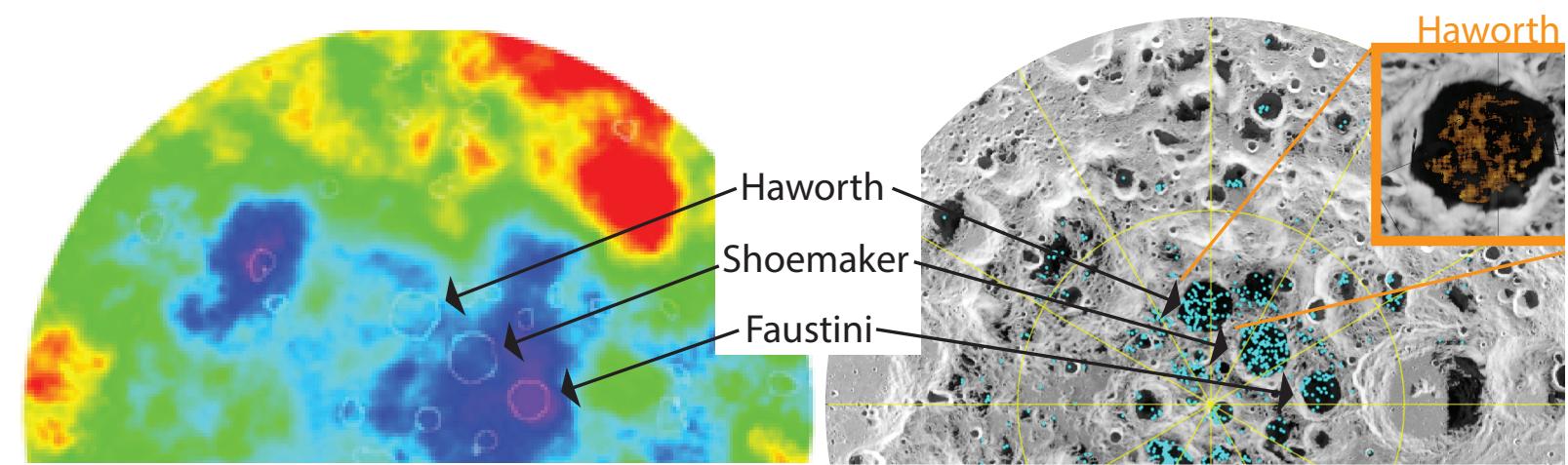

Figure 1. (Left) Water equivalent hydrogen in the top meter of regolith is determined from epithermal neutron flux by LEND on LRO [1] in the three large south polar PSRs: Haworth (51.4 km diameter, for scale); Shoemaker; and Faustini with blue colors corresponding to more H. (Right) Locations of water exposures on the surface determined from LAMP, LOLA, and $\mathrm{M}^{3}$ are shown in cyan [2]. The gray-scale background image indicates annual maximum temperature [3]. (Inset) LAMP data indicate surface frost within Haworth crater. PIPELiNE is a mission that would study the heterogeneity of volatile distributions shown here.

\section{Introduction}

A decade ago, a paradigm shift occurred in our thinking about water on the Moon and Mercury. We transitioned from wondering if water could exist on those airless bodies to the detection of ice in a lunar permanently shadowed region (PSR) [4], and more surprisingly, the observation of a widespread veneer of hydration on the sunlit surface of the Moon [5].

The ensuing decade brought observations from the MErcury Surface, Space ENvironment, GEochemistry, and Ranging (MESSENGER) mission of thick, pure ice deposits on Mercury [6-7]. Meanwhile, the Lunar Reconnaissance Orbiter (LRO) provided many observations to begin the characterization of lunar water. Surprisingly, in contrast to Mercury, lunar water appears more heterogeneously distributed and significantly less abundant than water on Mercury [8].

Even with this explosion of knowledge, fundamental questions remain unanswered. Whether the differences between the Moon and Mercury reflect different source fluxes, loss mechanisms, or ages of volatiles remains unknown. We lack fundamental knowledge regarding the source of volatiles, the age of volatiles, and the processes that modulate the abundance, distribution, and retention of water at the surface. Theoretical predictions and models are not sufficiently constrained by data and offer testable hypotheses. Acquiring new data and answering these questions will have profound implications for our understanding of the history of volatile availability in the inner solar system (and thus to Earth), the formation of the Moon and Mercury, and expectations for the presence of water on other airless bodies in the Solar System and on exoplanets.

The Moon's PSRs are the most accessible reservoir of solar system volatile history. Upcoming missions will provide further understanding of lunar volatiles, and a point from which to extrapolate to other celestial bodies. Orbital observations from small satellites will begin to hone the knowledge of the distribution of water on the sunlit and shadowed surfaces of the Moon. But what is really needed is ground truth. NASA's Commercial Lunar Payload Service (CLPS) program will bring experiments to the surface of the Moon to provide some ground truth of the present-day volatile cycle and the role of solar wind and meteoroids in the source or loss of water occurring on the Moon today. These are important pieces of the puzzle. The Volatiles Investigating Polar Exploration Rover (VIPER) mission will be groundbreaking. VIPER will be the first in situ investigation of multiple volatile trapping environments and is expected to provide new information about the abundance and distribution of volatiles in lunar polar regions.

We advocate for a mission in the next decade to reveal the composition, abundance, and distribution of volatiles deep within an old, cold PSR on the Moon and the relationship of 


\section{Mission to Characterize Volatiles in Old, Cold, Permanently Shadowed Regions}

those parameters to environmental drivers. One clear truth about lunar cold traps is that there is considerable heterogeneity in the composition and distribution of volatiles within a PSR and from one PSR to another as shown in Figure 1. The next giant leap in understanding volatile history and evolution can only come from exploring one of the coldest PSRs on the Moon, as the full history of volatiles is locked in the oldest, coldest $(<50 \mathrm{~K})$ PSRs. The PSRs accessible to short forays by a solarpowered rover like VIPER necessarily limited by proximity to solar power. This white paper describes a mission concept to go beyond the edges and to enter the depths of the Moon's large old PSRs with a mission called the Polar Ice Prospecting Explorer for Lunar No-light Environments (PIPELiNE).

\section{Science Objectives}

The cold traps in lunar PSRs have been caching an unparalleled scientific record of volatiles from many sources for billions of years. They likely contain volatiles from early lunar outgassing events, from asteroids and comets encountering the Earth-Moon system over time, and volatiles delivered to or liberated from the Moon continually via solar wind interactions and meteoroid bombardment. The measurement objectives of this mission concept are to sample volatile composition, abundance, physical form, and distribution in situ from an extremely old, cold lunar PSR and the environmental context of those parameters. These measurements would yield major contributions to the current state of knowledge in the field by: (a) providing critical ground-truthing of remotely sensed measurements that have thus far defined our understanding of lunar surface volatiles; (b) analyzing the driving processes that generate and modify the abundance, distribution, and composition of polar volatiles over time; (c) monitoring ongoing activity and transport of volatiles in the PSRs; (d) suggesting the source(s) and time history of inner solar system volatiles; and (e) characterizing the thermophysical and geotechnical properties of the cold-trapping environment.

These represent fundamental physical processes extending beyond the Moon to other solar system bodies and have been called out as critical topics in community documents (see Box 1) including the SCEM report [9], Advancing Science of the Moon (ASM-SAT) [10], Lunar Exploration Roadmap, LEAG Volatiles SATs 1 and 2 [11-12], Strategic Knowledge Gaps [13], and Visions and Voyages [14] and the Decadal midterm report [15]. It remains a high priority item for an investigation that can achieve significant breakthroughs in the coming decade.

\section{Science goals}

Determine composition, abundance, distribution, and physical form of lunar polar volatiles. DAS Characterize processes modulating retention and loss of volatiles. $D A$

Measure the ongoing activity and volatile transport at PSRs. DAS

Determine source(s) and time history of inner Solar System volatiles. DA

Provide ground-truth to remote sensing measurements. As

Determine the geotechnical properties of cold polar regolith. As

Characterize the space environment in PSRs. $s$

Compare contents and processes to Mercury, asteroids. $D$
Box 1

$\underline{\mathrm{KEY}}$

Linkage to

community guiding

documents

(D)ecadal Goal-V\&V; (A)SM-SAT Goal;

(S)trategic

Knowledge Gap

2.1 Ground-truthing of remotely sensed measurements. To date, most understanding of lunar polar volatile abundance, distribution, composition, and physical form is derived from remotely sensed observations of the Moon [2;4-5;16-21]. However, the spatial resolutions of these observations are substantially coarser than the likely scale over which lunar volatiles vary laterally and with depth. Figure 1 shows the distribution of hydrogen in the top meter of regolith from the LRO Lunar Exploration Neutron Detector (LEND) (left) and the surface frost from several remote sensing instruments superposed on a temperature map (right). While the measurements of ejecta probed by the Lunar Crater Observation and Sensing Satellite (LCROSS) may be within this scale, they only provide data 


\section{Mission to Characterize Volatiles in Old, Cold, Permanently Shadowed Regions}

for a single location on the surface. It is undetermined how representative these data are of the lunar poles. PIPELiNE will acquire in situ data to provide the critical ground-truthing required to better understand the existing database of polar remote sensing data.

2.2 Processes modulating volatile abundance, distribution, composition, and physical form. It is not well understood how the abundance, distribution, composition, and physical form of volatiles are altered after emplacement. A variety of factors may be important in understanding the physical and chemical alteration of lunar volatiles through time. Figure 2 illustrates how the distribution of volatiles may vary spatially in the lunar subsurface. The mode of volatile delivery is predicted to influence the volatile distribution. For example, volatiles delivered by a continuous mechanism at a lower rate should have a more homogeneous and diffuse distribution than volatiles delivered in episodic pulses. Impact gardening can produce spatial heterogeneities in volatile distribution because impacts remove volatiles via vaporization but also preserve volatiles through the emplacement of ejecta [22-23]. Additionally, the surface and near-surface thermal environments have a significant effect on volatile distribution, stability, and transport because the rates of diffusion and sublimation are modulated by temperature [24-30]. In situ molecular compositional measurements can inform our understanding of local modification processes that have altered volatiles, including impacts [22-23; 31], cosmic rays [32], solar wind sputtering [33], and dielectric breakdown [34]. Overall, understanding the processes that drive volatile modification will provide valuable insight into the implications of these processes. Subsequently, this understanding can be applied to volatiles on Mercury, asteroids, and other airless bodies in the solar system.

2.3 Activity and transport of volatiles. Complementary to understanding the processes that modify volatiles over long periods of time is witnessing the processes that are acting today. Recent results

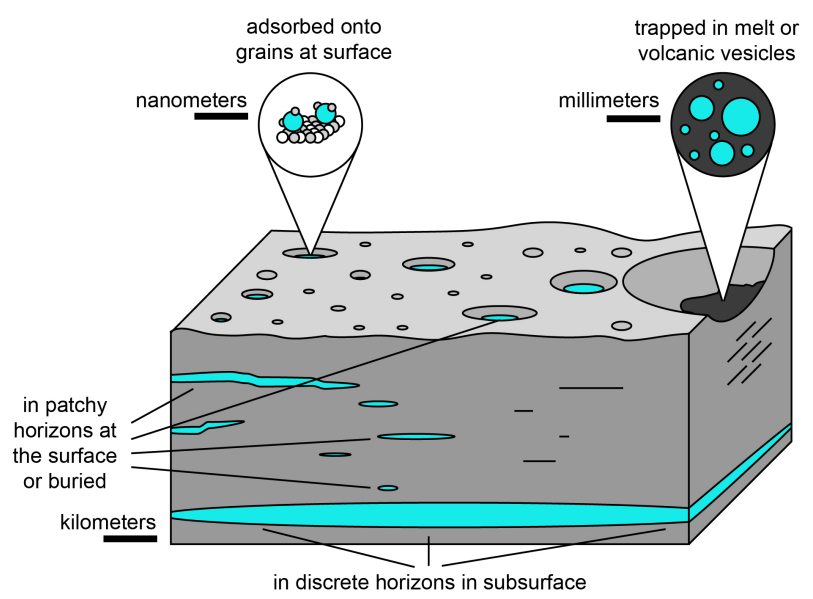

Figure 2. Water ice may exist on many lateral and depth scales in lunar polar regions [44]. from Lunar Atmosphere and Dust Environment Explorer (LADEE) imply the Moon is in a state of losing water [35]. In situ analysis could characterize the effects of different physical mechanisms that result in the addition or loss of volatiles. Measuring the present-day rates of mass change will reveal the efficiency of volatile transport on the Moon. Monitoring the surface volatile content daily, seasonally, and yearly can help to assess how dynamic the PSR volatiles are, which can be linked to exosphere, surface, and subsurface processes [23; 36-42]. In addition, assessing soil chemistry is essential to test transport models of lesser volatile elements such as $\mathrm{Zn}, \mathrm{S}$, and $\mathrm{Cl}[43]$.

2.4 Source and time history of volatiles. Both isotopic and chemical composition are key indicators of the original source of volatiles. However, direct compositional measurements of volatiles coldtrapped in lunar PSRs have been difficult to acquire due to viewing conditions in permanent darkness [2] and challenges in determining the composition of subsurface volatiles from orbit. $\mathrm{D} / \mathrm{H}$ ratios inform our understanding of the origin of water ice [45-47]. Sulfur content is a direct link to the contribution of volcanic outgassing to polar volatiles [48]. The presence of organics constrains asteroidal and cometary delivery sources [49-50]. Therefore, analyzing the present-day composition of volatiles will provide insight into the original sources of volatiles. 


\section{Mission to Characterize Volatiles in Old, Cold, Permanently Shadowed Regions}

Because modification processes acting in PSRs take time, spatial heterogeneity is related to the exposure age of the volatiles [51]. The distribution of volatiles with depth provides a window into the age and emplacement timing of volatiles. The physical form of the volatiles, whether they exist as ice blocks, pore-filling ice, adsorbed volatiles, or incorporated in the minerals (see Figure 2) is indicative of the source and time history. Quantification of the coherence of deposits and the relationship to thermal conditions will provide insights into the age of PSR volatiles beyond those provided by stratigraphy and superposition.

2.5 Thermophysical and geotechnical properties of PSRs. Many questions remain regarding the thermophysical and geotechnical properties of polar regolith. For example, regolith porosity of the uppermost surface appears to be higher inside PSRs than in illuminated regions [19; 52-54]; however, it is not clear what processes have generated the high porosity [34; 55-56]. A better understanding of the regolith porosity will improve our understanding of the electrostatic and thermophysical environment at the pole and will help quantify the pore-space available to cold-trapping. Characterizing the thermal and geotechnical properties of the lunar PSRs is also important in future ISRU. Therefore, better constraints on the bearing capacity [57] and the mechanical strength [58] will inform not only scientific objectives but also exploration priorities [13].

\section{Mission Implementation}

The PIPELiNE mission baseline is a nuclear-powered rover that will assay the volatile contents deep within the heart of a lunar PSR.

3.1 Mission Requirements. We already know that volatiles are heterogeneously distributed on the Moon [19;21; 2; 59-67]. Volatiles vary with depth, laterally, and from one PSR to another. Even an area that would appear to have high volatile content according to remote sensing data may have a patchy distribution on the surface and subsurface [22]. A static lander could sample a dry spot and not provide the data needed to characterize the composition and distribution of PSR volatiles. A mission that will robustly explore a larger area mitigates that risk. This imposes a requirement for mobility or for a distributed network of experimental packages.

Upcoming missions, including the VIPER mission, will explore polar volatiles and add to our knowledge. However, the large, old, cold PSRs are not accessible by a solar-powered rover such as VIPER. In order to sample the most volatile of the volatiles, the destination should be the coldest regions on the Moon, anticipated to be $<50 \mathrm{~K}$. Data indicate that the composition of volatiles on the surface changes at these low temperatures [21]. This drives PIPELiNE to require operation

Mission Design

Launch/Falcon 9

Direct injection at the Moon/Star-48

Landing using ALHAT directly in PSR

Landing site

Cold PSR (highly volatile compounds)

Positive detection of volatiles by remote sensing

Earth visibility

Traverse-friendly slopes

$\underline{\text { Rover }}$

Direct to Earth communications

MMRTG plus battery power

Flash illumination for navigation

Concept of Operations

Survey while roving to find location with high subsurface $\mathrm{H}$.

Perform site analysis with drill to sample at $10 \mathrm{~cm}$ depth increments: observe pilings with IR and ingest sample for composition analysis.

Include periods for recharging between traverse and sampling activities. in extremely cold environments.

Furthermore, we can consider that large impacts occurred long ago. In order to go back in history to sample volatiles emplaced and retained potentially for billions of years, one must go to an old PSR. While there exist some PSRs that could be reached by a series of forays from an illuminated region, the combination of the desire to sample hyper-volatiles and to assay volatiles that have the potential to be ancient leads us to target deep in permanent shadow, and subsequently the inability to use solar power for the mission. 


\section{Mission to Characterize Volatiles in Old, Cold, Permanently Shadowed Regions}

The PSR environment drives significant parts of the PIPELiNE design. Challenging topography and limited illumination conditions at the lunar poles preclude the exploration of such locations by solar-powered rovers [68]. A multi-mission radioisotope thermoelectric generator (MMRTG) is enabling for this mission in two ways. First, solar power would only allow short sorties on the order of $100 \mathrm{~m}$ into shadow, requiring frequent returns to sunlight for recharging. As shown in Figure 1, target study areas are inaccessible via 100-m sorties. A battery-only mission would require massive batteries, limiting payload capacity and mission duration [69]. In contrast, by using an MMRTG and battery, PIPELiNE is able to explore deep into PSRs with a mission duration of several months. A second advantage of the MMRTG system is that it naturally provides heat needed to maintain functionality of the spacecraft systems in the cold $(<100 \mathrm{~K})$ PSR environment.

3.2 Payload. Although alternate candidate payloads would add value to such a mission, one comprehensive payload at the Flagship cost level includes a gas chromatograph mass spectrometer (GCMS), neutron spectrometer (NS), Raman spectrograph (Raman), ground penetrating radar (GPR), infrared spectrometer (IRS), spatial heterodyne spectrometer (SHS), atmospheric neutral mass spectrometer (NMS), ion spectrometer (IS), traverse and context cameras (Cam), and a drill with temperature probes. These instruments are summarized in Table 1. At the New Frontiers cost cap, the payload will be smaller, perhaps to contain the GCMS, NS, IRS, Cam, and drill with embedded temperature sensors. Many of these systems are inherently active experiments (e.g., GPR, Raman). The dark environment of the PSR requires that PIPELiNE carry its own illumination for IRS, SHS, and Cam.

Table 1 PIPELiNE Strawman Payload

\begin{tabular}{|l|l|l|l|l|l|}
\hline Instrument & Mass (kg) & Peak Power & TRL & Function & Reference \\
\hline NavCams & 1 & $7.5 \mathrm{~W}$ & 9 & Navigation and context & {$[70]$} \\
\hline NS & 1.9 & $1.5 \mathrm{~W}$ & 6 & Volumetric H content & {$[71]$} \\
\hline GCMS & 15 & $60 \mathrm{~W}$ & 6 & Composition incl. isotopes & {$[72]$} \\
\hline IRS & 4 & $30 \mathrm{~W}$ & 6 & Comp., abundance, temperature & {$[71]$} \\
\hline Drill & 18.0 & $100 \mathrm{~W}$ & 6 & Subsurface access - 1 meter & {$[73]$} \\
\hline SHS & 1 & $6 \mathrm{~W}$ & 4 & Exosph. Composition \& isotopes & {$[74]$} \\
\hline Raman & 7.2 & $15.5 \mathrm{~W}$ & $4-6$ & Chemical \& isotopic compositions & {$[75]$} \\
\hline GPR & 6 & $10 \mathrm{~W}$ & 6 & Subsurface context, ice blocks & {$[76]$} \\
\hline IS & 5 & $9 \mathrm{~W}$ & 9 & Charged particle flux & {$[77]$} \\
\hline
\end{tabular}

3.3 Concept of Operations. While traversing, PIPELiNE will survey volatiles on the surface via IRS and in the subsurface via NS. Figure 3 shows a numerical simulation of an area representative of the PIPELiNE landing site, in which an ice sheet has been modified by impact gardening (bottom panel). The top panel presents how it would appear to a roving NS system [22]. The observations will indicate locations where the rover will stop and perform more detailed analysis of the volatiles. The detailed assessments will include drilling into the regolith, measuring the temperature as a function of depth, and analyzing the drillings via GCMS and IRS.

This system maps directly to the mission science objectives. (a) By systematically sweeping multiple areas of $100 \mathrm{~m}$ x $100 \mathrm{~m}$, PIPELiNE will provide ground-truth for remote sensing observations, which have spatial resolution on the order of $100 \mathrm{~m}$. (b) By determining the heterogeneity of the distribution of volatiles on the surface and subsurface and relating that to environmental conditions and models, PIPELiNE will identify the processes that modify the volatile contents of PSRs. (c) The NMS and SHS will monitor the local exosphere to quantify the present flux of volatiles in a PSR. (d) Direct detection of volatile composition, including isotopic analysis, via GCMS, Raman, and IRS will distinguish the roles of multiple sources in providing volatiles to the 


\section{Mission to Characterize Volatiles in Old, Cold, Permanently Shadowed Regions}
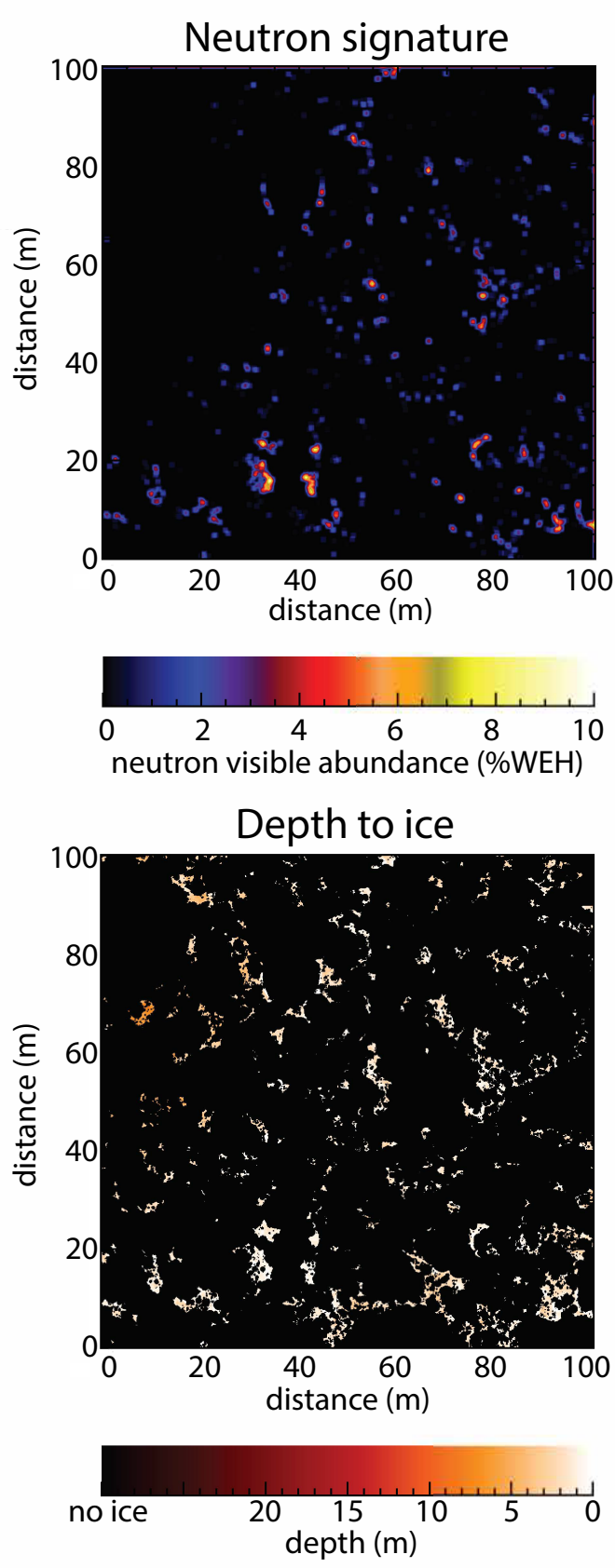

Figure 3. A model shows the ice distribution in a $100 \mathrm{~m} \times 100 \mathrm{~m}$ area after $2 \times 10^{9}$ years of impact gardening has acted on a $10 \mathrm{~cm}$ thick ice sheet. (Top) simulated NS measurements; (bottom) depth (in meters) of drilling required to access ice.
PSR. (e) Data from the drill and rover mobility system indicate thermal and geotechnical properties of the PSR, as do images of the rover track and regolith structure.

\section{Next Steps}

4.1 Need for a mission concept study. Previous work has been done to define a mission concept for a PSR rover that came in very close to the New Frontiers cost cap [69]. Furthermore, development of the VIPER mission can be leveraged. Adding the expenses associated with the thermal environment of the coldest PSRs and using a nuclear power system to the VIPER mission suggests that exploration deep into the past is possible under New Frontiers in the next decade. However, a mission design exercise should be conducted to confirm the cost and to define the science that can be accomplished within New Frontiers.

The mission design exercise should examine a VIPER-like mission and modifications needed, including landing in the dark, thermal, and power. Trade studies should be conducted on direct-to-Earth (DTE) communications vs. using a communications relay. Additional measurements should be considered. Also, there should be some consideration of alternative architectures including a set of penetrometers, or multiple LCROSS-like impact experiments.

4.2 Programmatic Considerations. In addition to the science discussion above, water ice on the Moon is highly relevant from commercial and exploration perspectives. Water ice on the Moon can be exploited as a resource, and whether it is commercially viable depends on the abundance, distribution, composition, and accessibility. While volatiles in cold deep PSRs may be less accessible, their abundance and/or distribution may be beneficial for harvesting and/or storing extracted volatiles. This substantial crossover between pure science and applied science is rather unique programmatically. The fraught history of

VIPER (previously Resource Prospector and previously of HEOMD) demonstrates the potential programmatic issues that such a mission can have when it is interdisciplinary. NASA SMD has decided to remove Planetary Defense missions from the competed mission lines because of the programmatic issues. Resource missions may also be better handled as directed missions and removed from the competed lines. If this is done, every effort should be made to involve both the science and resource communities in the mission definition to ensure meeting both science and resource goals. 


\section{Mission to Characterize Volatiles in Old, Cold, Permanently Shadowed Regions}

Another consideration is how to factor CLPS deliveries into the cost calculation for lunar missions. CLPS task orders include both the launch vehicle and lunar lander. The way to account for a CLPS delivery should be described in competed mission calls.

\section{References}

[1] McClanahan, T. P. et al. (2018) LPSC 2339; [2] Li, S. et al. (2018) PNAS 115, 8907-8912; [3] Williams, J.-P. et al. (2019) JGR-P 124, 2505-2521; [4] Colaprete, A. et al. (2010) Science 330, 463468; [5] Pieters, C.M. et al. (2009) Science 326, 568-572; [6] Neumann, G. A. et al. (2013) Science 339: 296; [7] Chabot, N. L. et al. (2014) Geology 42(12): 1051-1054; [8] Lawrence, D. J. (2017) JGR-P 122(1): 21-52; [9] Paulikas, G.A., et al. (2007) The Scientific Context for the Exploration of the Moon; [10] Lawrence, S. et al. (2018) LEAG ASM-SAT report; [11] Lucey, P.G., et al. (2014) LEAG Volatiles (VSAT) Report; [12] Hurley, D.M. et al. (2017) LEAG Volatiles (VSAT2) Report; [14] Squyres, S.W. et al. (2011) https://doi.org/10.17226/13117; [15] House, C.H. et al. (2017) https://doi.org/10.17226/24843 ; [16] Haruyama, J. et al. (2008) Science 322(5903): 938-939; [17] Clark, R.N. (2009) Science 326, 562-564; [18] Mitrofanov, I.G., et al. (2010) Science 330, 483-486; [19] Gladstone, G.R. et al. (2012) JGR-P 117, E00H04; [20] Hayne, P.O. et al. (2015) Icarus 255, 58-69; [21] Fisher, E.A. et al. (2017) Icarus 292, 74-85; [22] Hurley, D.M. et al. (2012) GRL 39, L09203; [23] Costello, E.S. et al. (2019) LPSC 50, abstract 1991; [24] Paige, D.A. et al. (2010) Science 330, 479-482; [25] Hibbitts, C.A. et al. (2011) Icarus 213, 64-72; [26] Poston, M.J. et al. (2013) JGR-P 118, 105-115; [27] Poston, M.J. et al. (2015) Icarus, 255, 24-29; [28] Schorghofer, N., Aharonson, O. (2014) ApJ 788, 169; [29] Siegler, M., et al. (2015) Icarus 255, 78-87; [30] Siegler, M.A. et al. (2016). Nature 531, 480-484; [31] Stopar, J.D. et al. (2018) PSS 162,157-169; [32] Crites, S.T. et al. (2013) Icarus 226, 1192-1200; [33] Zimmerman, M.I., et al. (2013) Icarus, 226(1) 992-998; [34] Jordan, A.P. et al. (2015) JGR-P 120, 210-225; [35] Benna, M. et al. (2019) Nature Geo., 12(5), 333338; [36] Crider, D.H., Vondrak, R.R., (2002) ASR 30, 1869-1874; [37] Sunshine, J.M. et al. (2009) Science 326, 565-568; [38] Li, S., Milliken, R.E. (2017) Science Adv. 3, e1701471; [39] Schorghofer, N. et al. (2017) Icarus 298, 111-116; [40] Bandfield, J.L. et al. (2018) Nature Geo. 11, 173; [41] Prem, P. et al. (2018) Icarus 299, 31-45; [42] Grumpe, A. et al. (2019) Icarus 321, 486-507; [43] McCubbin, F.M. et al. (2015) Amer. Min. 100, 1668-1707; [44] Needham, D.H., Kring, D.A., (2018) Lunar Polar Volatiles Workshop; [45] Arnold, J.R. (1979) JGR 84, 5659-5668; [46] Schorghofer, N. (2014) GRL 41, 4888-4893; [47] Marty, B. et al. (2016) EPSL 441, 91-102; [48] Needham, D.H., Kring, D.A., (2017) EPSL 478, 175-178; [49] Zhang, J.A., Paige, D.A. (2009) GRL 36, L16203; [50] Lucey, P.G. (2000) Proc. SPIE 4137; [51] Deutsch, A.N. et al. (2019) LPSC 50, abstract 1150; [52] Schultz, P.H. et al. (2010) Science, 330(6003), 468-472; [53] Mandt, K.E. et al. (2016) Icarus 273, 114-120; [54] Byron, B.D. et al. (2019) LPSC 50, abstract 3115; [55] Chen, K. et al. (2006) Nature 442, 257; [56] Farrell, W.M. et al. (2010) doi.org/10.1029/2009JE003464; [57] Sargeant, H.M. et al. (2010) LPSC, 1792; [58] Gertsch, L. et al. (2008) Int. Conf. on Case Histories in Geotech. Eng., 2; [59] Sanin, A.B. et al. (2017) Icarus 283: 20-30; [60] Spudis, P.D. et al. (2013). JGR-P 118(10): 20162029; [61] Miller, R.S. et al. (2014) Icarus 233: 229-232; [62] Neish, C. D. et al. (2011) JGR-P 116(E1): E01005; [63] Shearer, C.K., et al. (2016) LEAG GAP-SAT; [64] Elphic, R. C. et al. (2007) doi:10.1029/2007gl029954; [65] McClanahan, T. P. et al. (2015) Icarus 255: 88-99; [66] Thomson, B. J. et al. (2012) GRL 39(14): L14201; [67] Patterson, G.W. et al. (2017) Icarus, 283, 2-19; [68] Flahaut, J. et al. (2020) PSS 180, 104750; [69] Shearer, C. et al. (2011) LPVE mission concept study; [70] Maki J. et al. (2012) Space Sci. Rev. 170, 77-93; [71] Ennico-Smith, K. et al. (2020) LPSC 2898; [72] Mahaffy, P.R. et al. (2012) SSR 170, 401-478; [73] Zacny, K. et al (2018) Space Resources Roundtable; [74] Hosseini, S. et al. (2018) COSPAR B3.1-82-18; [75] Retherford, K.D. et al. (2019) LPSC; [76] Hamran, S.E. et al. (2015) IWAGPR; [77] Delcourt, D., et al. (2016) JGR-SP 121, 6749_ 6762. 